$15^{\text {th }}$ International Conference on

AEROSPACE SCIENCES \& AVIATION TECHNOLOGY,

$\boldsymbol{A S A T}$ - 15 - May 28 - 30, 2013, Email: asat@ mtc.edu.eg, Military Technical College, Kobry Elkobbah, Cairo, Egypt, Tel: +(202) 24025292 -24036138, Fax: +(202) 22621908

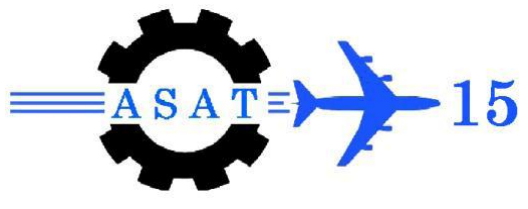

\title{
Parametric Study of Periodic Structures
}

\author{
Somaia Talaat ${ }^{*}$, M. Tawfik ${ }^{\dagger}$, H, M. Negm ${ }^{\dagger}$
}

\begin{abstract}
In the past decades, many numerical and experimental investigations on the capabilities of periodic structures for vibration attenuation and band localization were performed, and proved those capabilities for different simple structures. Earlier investigation of such phenomenon in periodic plates was not promising due to different factors. A parametric study is a systematic way to vary a number of model parameters and have the system run for one or several analyses for each combination of parameters. In this research, investigation of the effect of varying different parameters on the attenuation bands location and width of four different patterns of periodic plates will be discussed.
\end{abstract}

Keywords: Periodic structure, attenuation (stop) curves, propagation factor

\section{Nomenclature}

$\mu \quad$ Propagation Factor

$\mu_{\mathrm{x}} \quad$ Propagation Factor in $\mathrm{x}$-direction

$\mu_{\mathrm{y}} \quad$ Propagation Factor in y-direction

A Attenuation in the vibration amplitude between the input and the output

B Phase difference between the input and the output vibration wave

\section{Introduction}

Over the last decades, research on effective methods for attenuating vibration levels in structures has been very active. The periodic structure technique is one of these effective methods which has a lot of applications especially in the aerospace industry, such as satellite solar panels, aircraft fuselages and wings. A periodic structure consists fundamentally of a number of identical structural components which are joined together to form the whole structure. Discontinuities which can be induced with various forms such as masses, cross sectional geometries, and changes in material, do cause reflections of the vibration wave that may reduce the transmitted energy. Yet the degree of attenuation is weak over a broad range of frequencies, and the regions between these discontinuities exhibit local resonances.

The existence of complete band gaps and resonances in a plate with a periodic stubbed surface was demonstrated numerically and experimentally by Wu et al [1]. It was demonstrated that as the stub height approaches about triple the plate thickness a complete narrow band gap develops. The vibration control of a cantilever aluminum plate using a periodic configuration of RL (resistive-inductive) shunted piezoelectric patches was investigated in [2]. Numerical and experimental analyses were performed to 4-node Kirchhoff plate elements, and a reverse

Space Division, National Authority of Remote Sensing and Space Sciences, Cairo, 11769, Egypt.

${ }^{\dagger}$ Aerospace engineering Department, Cairo University, Giza, 12613, Egypt. 
approach was used for the finite element analysis. Unit cell analysis showed that increasing the shunting resistance reduces the attenuation but increases the region of effectiveness.

Experimental analyses demonstrated that the tuning capabilities of the RL networks can be effectively used within a periodic framework to obtain a broadband control effect. Both numerical and experimental results were encouraging in the sense that a unit cell approach is capable of accurately predicting the actual response of the system.

The vibration characteristics of a plate with shunted piezoelectric patches were also investigated by Tawfik [3]. The response was investigated when the patches were periodically distributed. He formulated a plate spectral finite element model and compared the results with experimental results, which showed reasonable agreement. Later numerical and experimental investigations of the application of a periodic array of resistive-inductive (RL) shunted piezoelectric patches for the attenuation of broadband noise radiated by a flexible plate in an enclosed cavity was investigated [4]. That research showed that the tuning capabilities of simple RL shunt circuits were combined with the filtering characteristics of periodic structures to obtain a tunable periodic plate, and the resulting smart structure experienced significant reductions in its noise radiation capability. Numerical simulations demonstrated the applicability of Bloch theorem [5] for unit-cell analysis on the in vacuo plate [6] as a tool to predict the frequency ranges of effective structural acoustic control. Also, experiments were performed to validate the numerical predictions, and demonstrate the effectiveness of the proposed strategy.

Recently a thin epoxy plate containing a periodic square array of lead discs hemmed around by rubber was considered [7]. The structure's response was calculated theoretically using periodic expansion of Bloch theorem. Numerical results showed that full band gaps of flexural vibration do exist in the thin plate. It was also shown that the width of the first full band gap is about three times larger than that in a thin steel plate with $2 \mathrm{D}$ binary locally resonant structures. The effect of the lattice constant [7] and thickness of the plate on the band gap have also been demonstrated. Casimir and Kevorkian [8] illustrated the principle of developing two dimensional elements such as plates, and showed how symbolic computation software could be used in this purpose. The method of construction of dynamic stiffness matrices was applied to rectangular Kirchhoff continuous elements, and a study of harmonic response over a large frequency range was made. It offers a clear advantage over finite elements, particularly its high precision and low memory cost. In Ref. [8] the effect of periodicity on the frequency response of different forms of periodicity was also investigated.

An analytical model that incorporates an infinite number of periodically spaced masses into the equations of elasticity that model motion and stress in a two-dimensional fully elastic solid was studied by Hull [9]. The examination of a periodic plate where the periodicity is presented by masses on the edge of the plate and where the masses reside within the interior of the plate was studied by Hull in this paper. The solution of an elastic plate harmonically loaded in space and time containing periodic edge masses at a time and embedded masses at another time has been derived and compared favorably with previously developed thin plate models at low frequencies. Also a numerical and analytical model of a novel design of a sandwich plate based on the antagonistic approach, and an application of SMA elements, were presented [10]. A two-dimensional model of the flexure of a thin plate reinforced with periodic families of separated thin rods, symmetrical about the middle plane, was analyzed in [11]. 


\section{Objective}

A parametric study is a systematic way to vary a number of model parameters and have the system run for one or several analyses for each combination of parameters. Here in this research investigation of the effect of varying different parameters on the attenuation bands location and width of four different patterns of periodic plates will be introduced as an extension of the work of Xiao [7]. The study was intended to attenuate or delay the vibration in certain bands of frequency. As the design moves forward one needs to assess the impact of changing certain parameters on the design. The parameters can include dimensional and geometric parameters. Parametric studies allow to nominate parameters for evaluation, define the parameter range, specify the design constraints, and analyze the results of each parameter variation. For this reason a study of the effect of varying the following parameters on the frequency response of periodic plate structures will be carried out in the following sections:

- Configuration of periodicity.

- Thickness ratio.

- Aspect ratio of the periodic cell.

\section{Configuration of Periodicity}

The capability of certain periodic configurations in attenuating vibration was investigated experimentally and numerically in [12]. Figure 1 shows the different patterns of these periodic configurations, while Figure 2 shows the cells of the four periodic structures. For each cell configuration the effect of thickness ratio and the aspect ratio variation on the vibration attenuation band widths will be studied in the following sections.
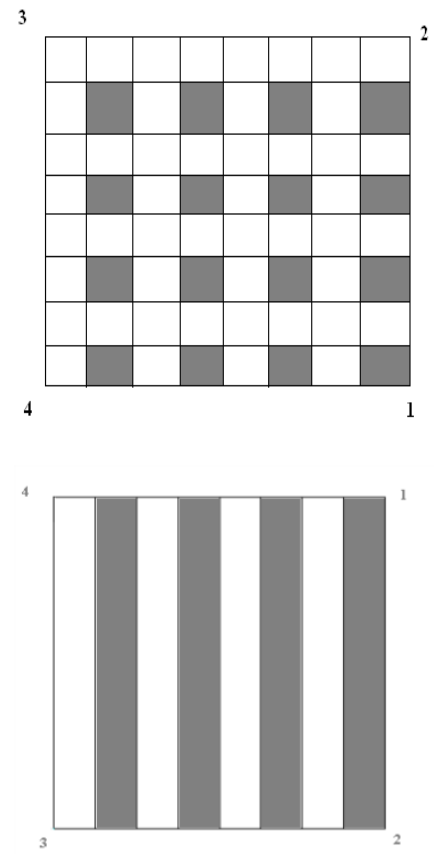
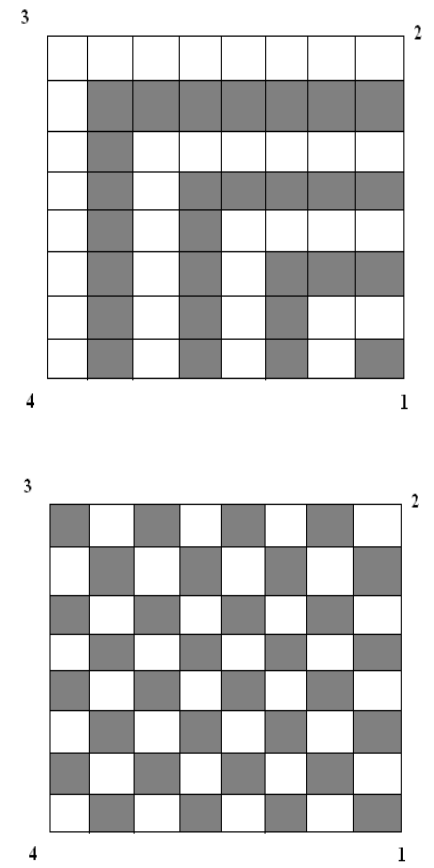

Figure 1 Four different periodic structures 


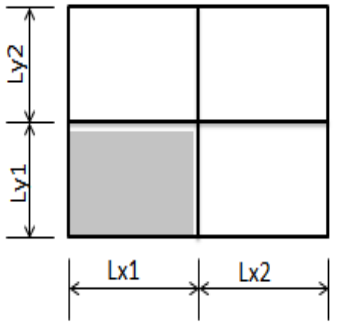

a- Cell Configuration 1

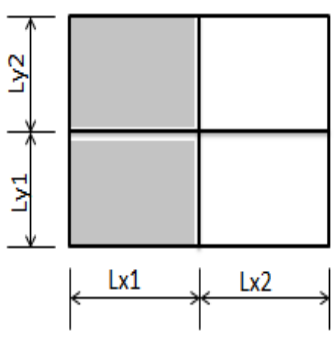

b. Cell Configuration 2

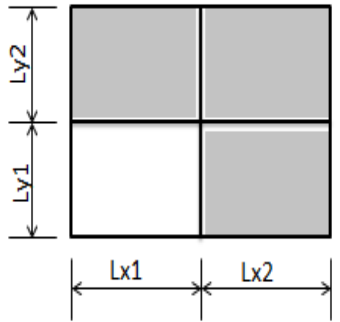

c. Cell Configuration 3

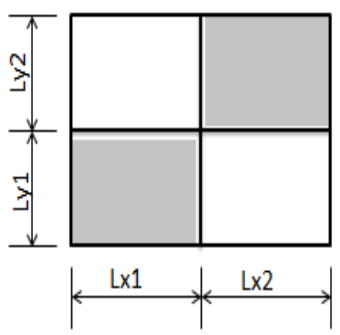

d- Cell Configuration 4

Figure 2 Four different cell configurations

\section{Effect of changing thickness ratio on the structural dynamic behavior of configuration cell \#1}

In this section the effect of varying the thickness ratio of the cell structure on its dynamic behavior will be studied. Consider cell configuration \#1, with variable thickness ratio ranging from 0.2 to 5 . The thickness ratio is $t_{2} / t_{1}$, where $t_{1}$ is the thickness of the grey element of the cell, and $t_{2}$ is the thickness of the other three elements.

Figure 4 shows the effect of varying the thickness ratio $\left(t_{r}\right)$ on the lower and upper bounds of the first stop band of cell configuration \#1 at angle $45^{\circ}$. This figure shows that as the thickness ratio increases the first attenuation band is shifted to higher frequencies.

Figure 5 shows the effect of varying the thickness ratio on the first stop band width. It shows that as the thickness ratio increases the attenuation band becomes wider, with a near linear variation for thickness ratios between 3.4 and 5 .

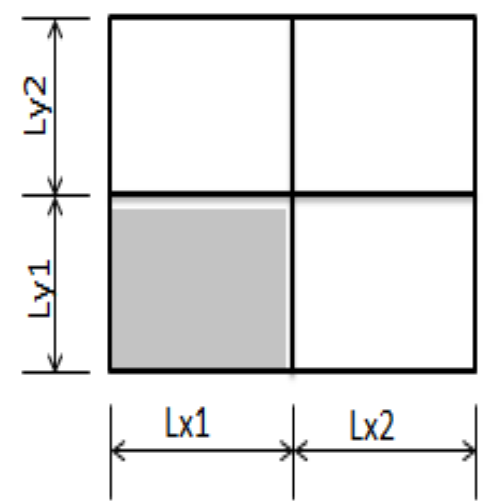

Figure 3 Cell Configuration \#1 


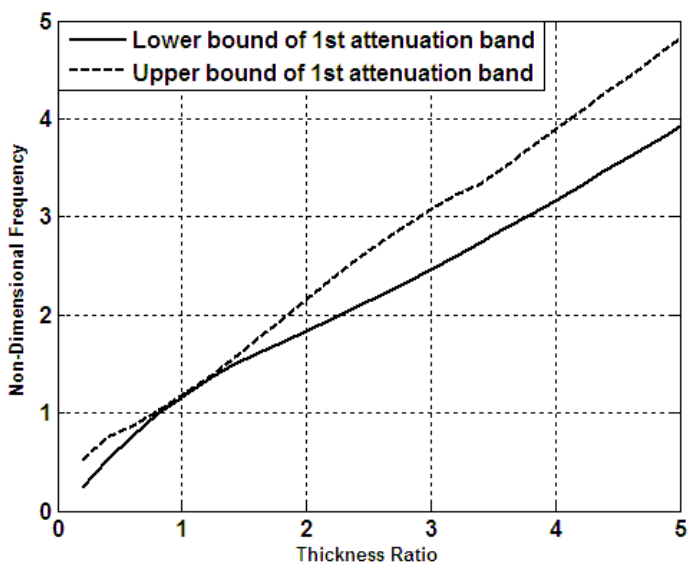

Figure 4 Lower and Upper bounds variation with thickness ratio of cell configuration \#1 at angle $45^{\circ}$

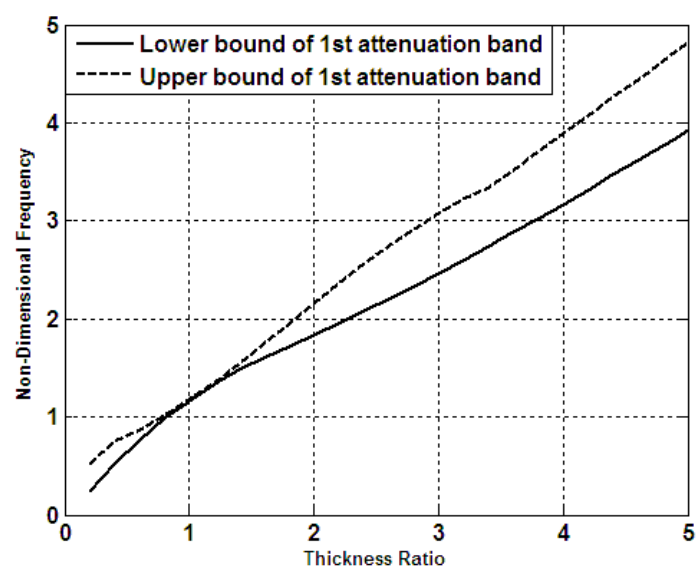

Figure $51^{\text {st }}$ stop band width variation with thickness ratio of cell configuration $\# 1$ at angle $45^{\circ}$

\section{Effect of changing aspect ratio of configuration cell \#1}

The cell aspect ratio has a significant effect on the location and width of the attenuation bands. The effect of changing aspect ratio on the first, second and third attenuation bands is shown in Figure 6 to Figure 9 with propagation factors $\mu=\alpha+0$ and $\mu=\alpha+i \beta$.

Figure 6 shows the lower and upper bounds of the first, second, and third attenuation bands of cell configuration \#1 with black, green, and blue lines respectively. Following the lower and upper bounds of the first attenuation band we see that the first attenuation band divides into two bands at aspect ratio 3 and the green lines coincide with the blue lines at that ratio, which means that the second band becomes the third attenuation band. The same is observed with a propagation factor with an imaginary part equal to $\beta$. The first, second, and third attenuation bands are plotted with black, green and blue lines respectively in Figure 7. The figure shows at which aspect ratios the attenuation bands merge or separate.

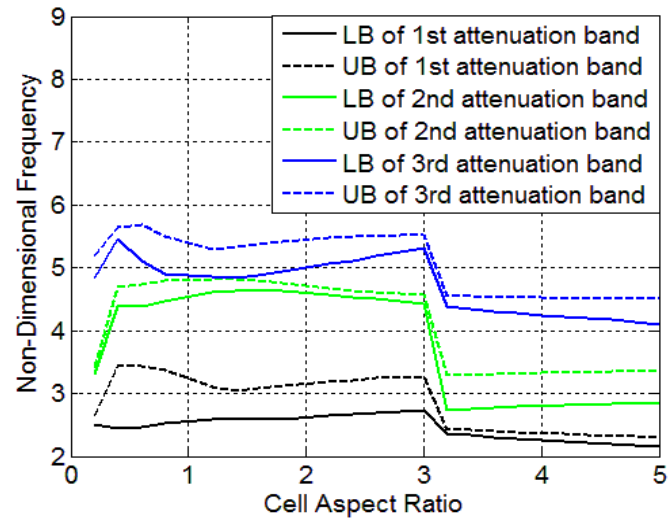

Figure 6 Lower and Upper bounds variation with aspect ratio of cell configuration \#1 at angle $45^{\circ}$ for propagation factor $\mu=\alpha+0$

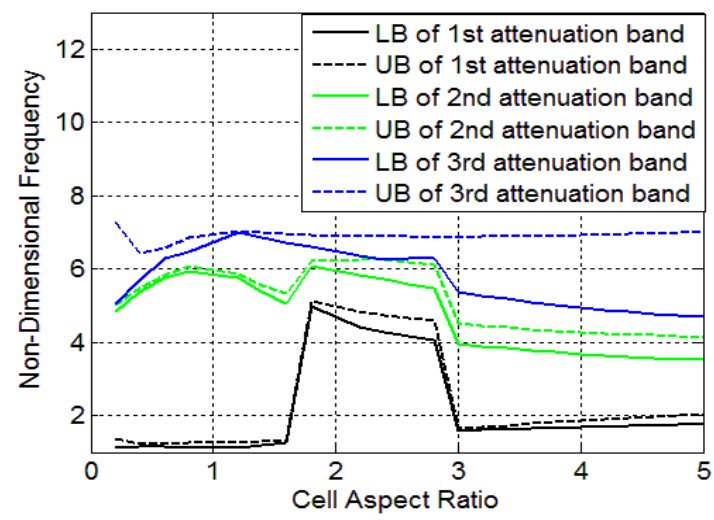

Figure 7 Lower and Upper bounds variation with aspect ratio of cell configuration \#1 at angle $45^{\circ}$ for propagation factor $\mu=\alpha+i \beta$ 
Figure 8 and Figure 9 show the variation of the attenuation bands width with the cell aspect ratio for a real and a complex propagation factor.

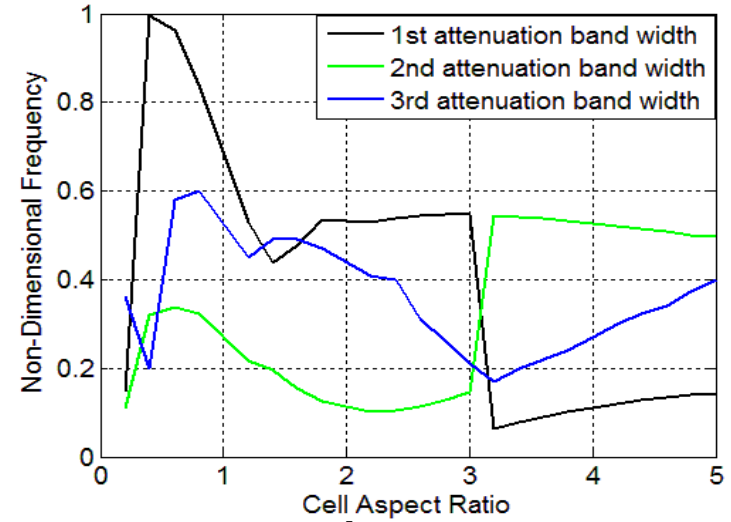

Figure $81^{\text {st }}, 2^{\text {nd }}$ and 3rd stop band width variations with aspect ratio of cell configuration \#1 at angle $45^{\circ}$ for propagation factor

$$
\boldsymbol{\mu}=\boldsymbol{\alpha}+\mathbf{0}
$$

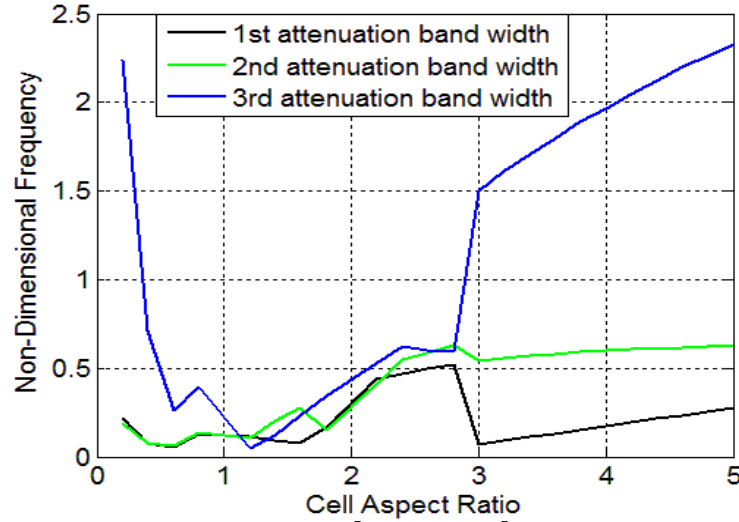

Figure $91^{\text {st }}, 2^{\text {nd }}$ and $3^{\text {rd }}$ stop band width variations with aspect ratio of cell configuration $\# 1$ at angle $45^{\circ}$ for propagation factor

$$
\mu=\alpha+i \beta
$$

\section{Effect of changing thickness ratio on the structural dynamic behavior of configuration cell \#2}

The second configuration is the most common configuration, and can be found in many life applications. Figure 10 shows the configuration of the second cell with two thick elements and two thin elements. For equal length and width the effect of changing the thickness ratio from 0.2 to 5 on the cell dynamic behaviour was investigated. Figure 11 shows the effect of changing thickness ratio on the lower and upper bounds of the first attenuation band while Figure 12 shows that the first attenuation band width increases as the thickness ratio increases.

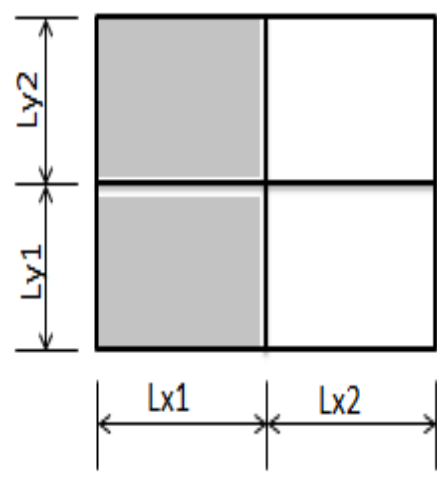

Figure 10 Cell Configuration \#2 


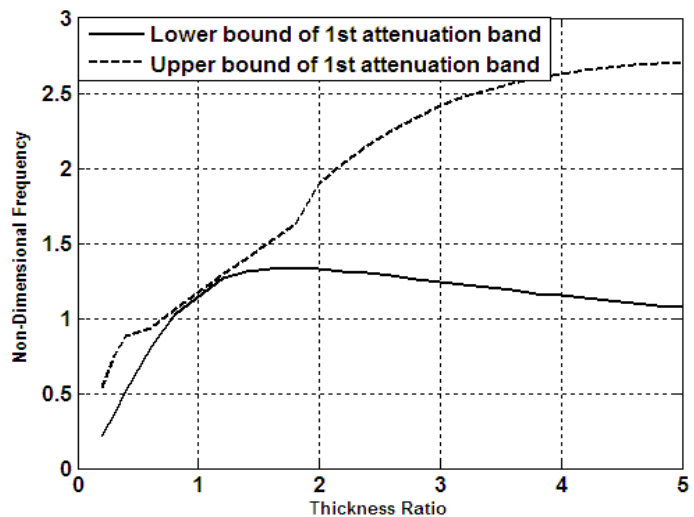

Figure 11 Lower and upper bounds variation with thickness ratio of cell configuration \#2 at angle $45^{\circ}$

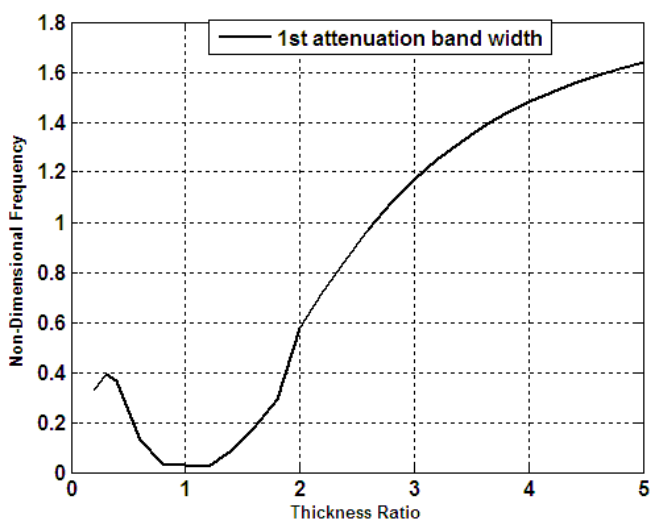

Figure $121^{\text {st }}$ stop band width variations with thickness ratio of cell configuration $\# 2$ at angle $45^{\circ}$

\section{Effect of changing aspect ratio of configuration cell \#2}

The effect of changing the cell aspect ratio between 0.2 and 5 on the dynamic behaviour of the periodic structure while keeping the thickness ratio constant at 0.5 will now be investigated.

The start and the end of the first, second, and third attenuation bands are plotted in Figure 13 to show the effect of aspect ratio variation on the attenuation bands location. The figure shows that at aspect ratio 2 the first attenuation band is split into two attenuation bands.

Figure 14 shows the change in the lower and upper bounds of the first, second and third attenuation bands with the variation of the aspect ratio for a propagation factor $\mu=\alpha+i \beta$. The figure shows that the first attenuation band is split into two attenuation bands at aspect ratio 2.2 , which then remerge again at aspect ratio 4 .

Figure 15 and Figure 16 show the effect of changing the aspect ratio on the first, second, and third stop bands widths for a propagation factors $\mu=\alpha+0$ and $\mu=\alpha+i \beta$ respectively.

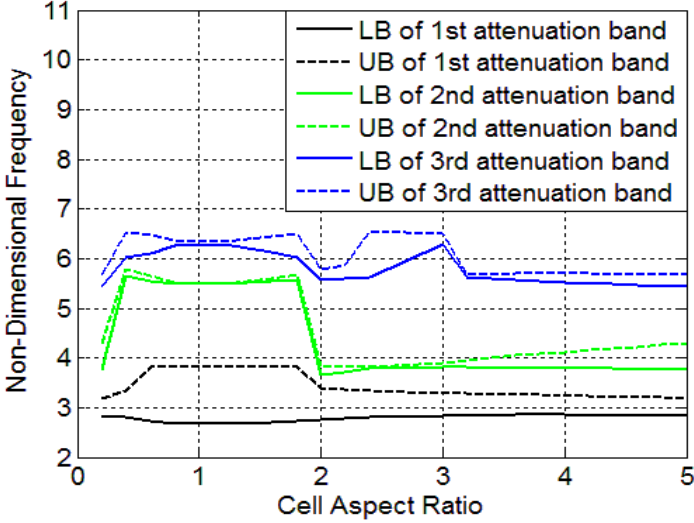

Figure 13 Lower and upper bounds variation with aspect ratio of cell configuration \#2 at angle $45^{\circ}$ for propagation factor $\mu=\alpha+0$

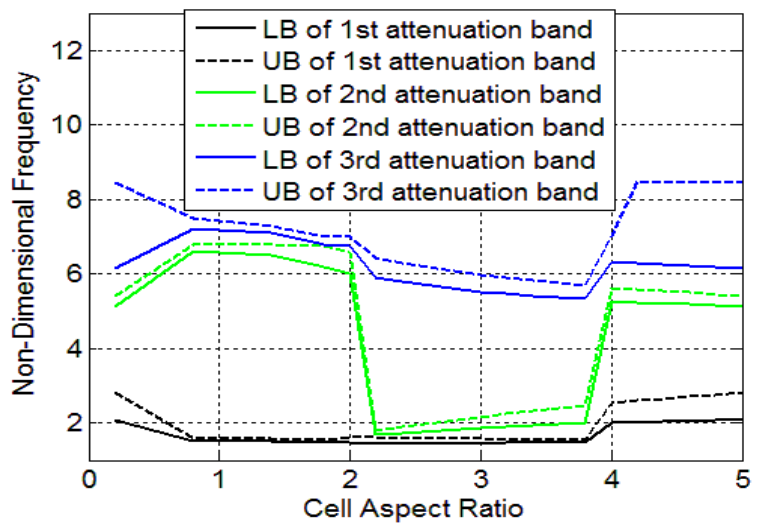

Figure 14 Lower and upper bounds variation with Length ratio of cell configuration \#2 at angle $45^{\circ}$ for propagation factor $\mu=\alpha+i \beta$ 


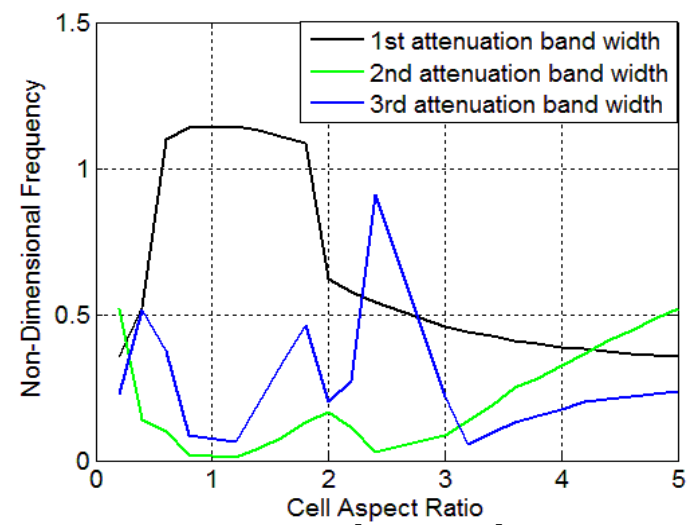

Figure $151^{\text {st }}, 2^{\text {nd }}$ and $3^{\text {rd }}$ stop band width variations with length ratio of cell configuration \#2 at angle $45^{\circ}$ for propagation factor

$$
\boldsymbol{\mu}=\boldsymbol{\alpha}+\mathbf{0}
$$

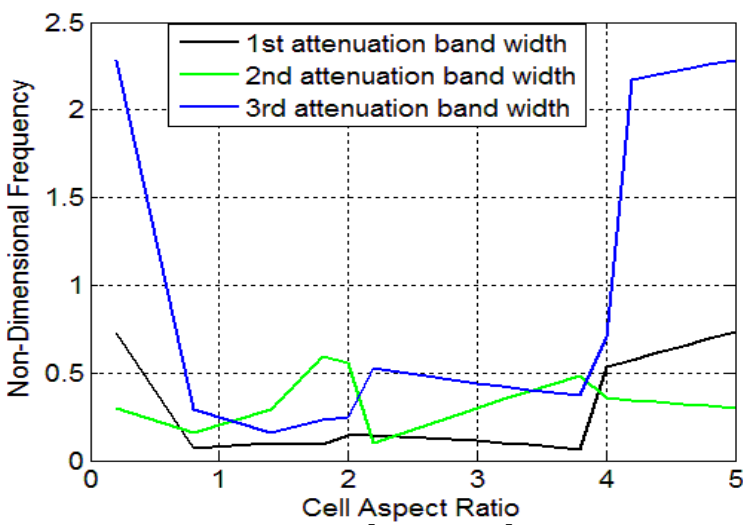

Figure $161^{\text {st }}, 2^{\text {nd }}$ and $3^{\text {rd }}$ stop band width variations with Length ratio of cell configuration \#2 at angle $\mathbf{4 5}^{\circ}$ for propagation factor

$$
\mu=\alpha+i \beta
$$

\section{Effect of changing thickness ratio on the structural dynamic behavior of configuration cell \#3}

Figure 17 shows the third configuration of the periodic cell. We shall investigate the effect on its dynamic behaviour of the variation of the cell thickness ratio and aspect ratio. In this section the effect of changing the thickness ratio on the location and width of the first attenuation band will be studied.

Figure 18 shows the lower and upper bounds plotted versus the non-dimensional frequency of the first attenuation band due to thickness ratio variation. Figure 18 shows that the first attenuation band is shifted to higher frequencies as the thickness ratio increases up to the thickness ratio 2. Then the first attenuation band is shifted to lower frequencies as the thickness ratio increases further.

Figure 19 shows the first stop band width variation with the thickness ratio. This figure shows that the band width decreases as the thickness ratio becomes closer to 1 , and then increases after that.

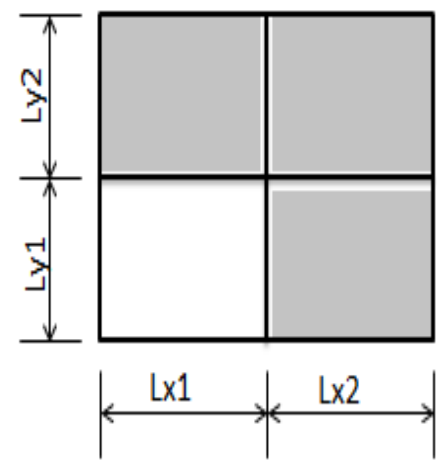

Figure 17 Cell Configuration \#3 


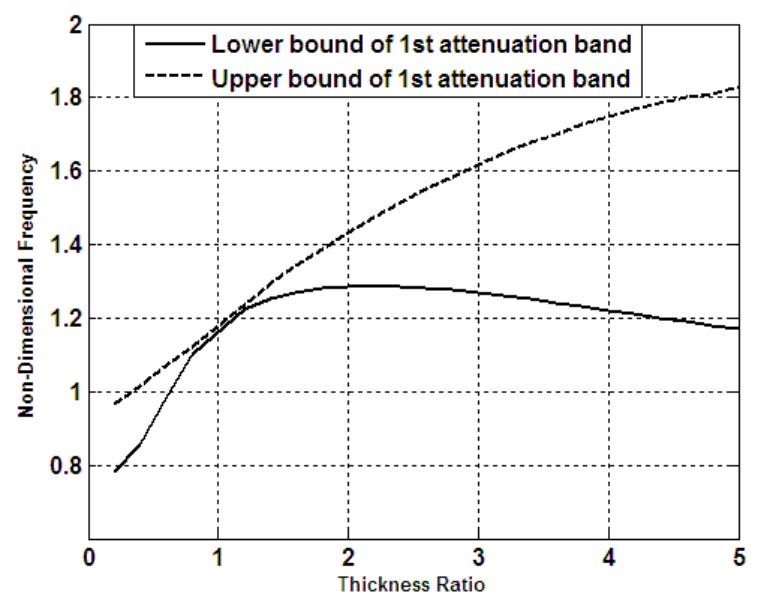

Figure 18 Lower and upper bounds variation with thickness ratio of cell configuration $\# 3$ at angle $45^{\circ}$

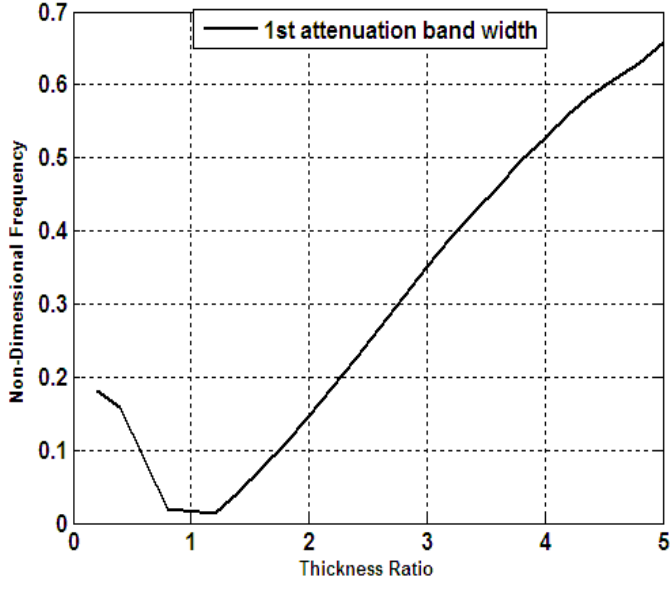

Figure $191^{\text {st }}$ stop band width variations with thickness ratio of cell configuration $\# 3$ at angle $45^{\circ}$

\section{Effect of changing aspect ratio of configuration cell \#3}

Figure 20 shows the results of the investigation. Changing the aspect ratio from 0.2 to 0.4 , the first attenuation band becomes smaller and moves near to the second attenuation band. Figure 20 shows a jump in the bands location when the cell aspect ratio changes from 0.2 to 0.4 . The next jump occurs from ratio of 2 to ratio of 2.2 .

Figure 21 shows the effect of changing the aspect ratio on the location of the first, second, and third attenuation bands with a propagation factor $\mu=\alpha+i \beta$. It shows that the first attenuation band divides into two attenuation bands in the range from 1.4 to 1.6 of the aspect ratio, and then they merge again.

Figure 22 and Figure 23 show the first, second, and third attenuation bands width variation with the aspect ratio with propagation factors $\mu=\alpha+0$ and $\mu=\alpha+i \beta$ respectively.

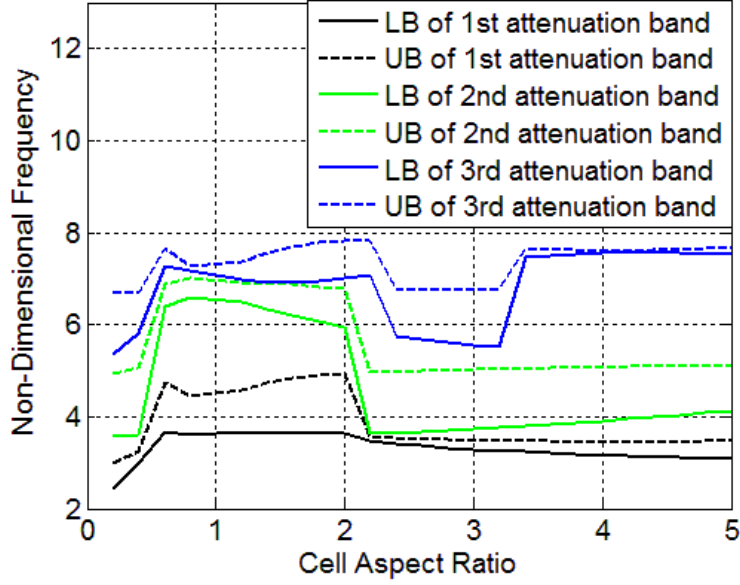

Figure 20 Lower and upper bounds variation with length ratio of cell configuration \#3 at angle $45^{\circ}$ for propagation factor $\mu=\alpha+0$

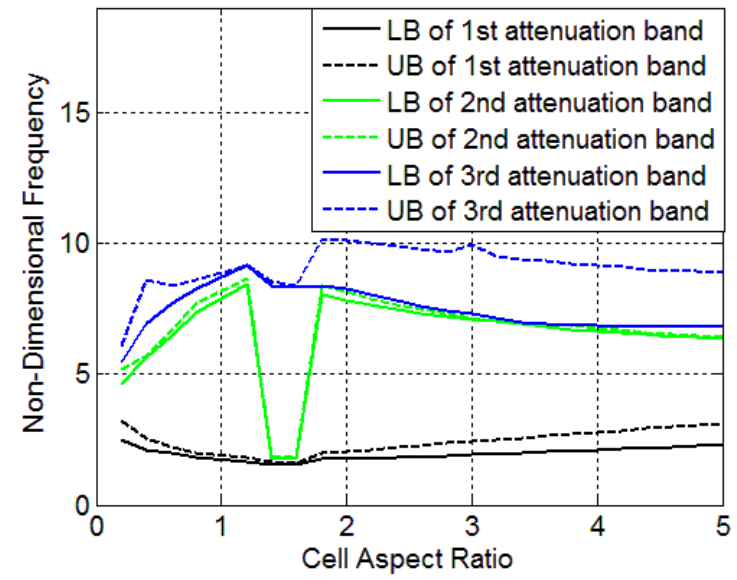

Figure 21 Lower and upper bounds variation with length ratio of cell configuration \#3 at angle $45^{\circ}$ for propagation factor $\mu=\alpha+i \beta$ 


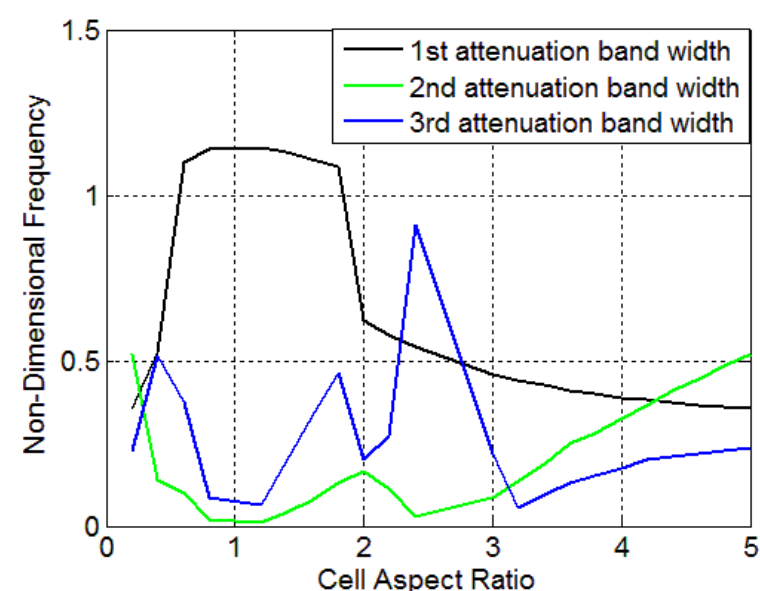

Figure $221^{\text {st }}, 2^{\text {nd }}$ and $3^{\text {rd }}$ stop band width variations with length ratio of cell configuration \#3 at angle $45^{\circ}$ for propagation factor

$$
\boldsymbol{\mu}=\boldsymbol{\alpha}+\mathbf{0}
$$

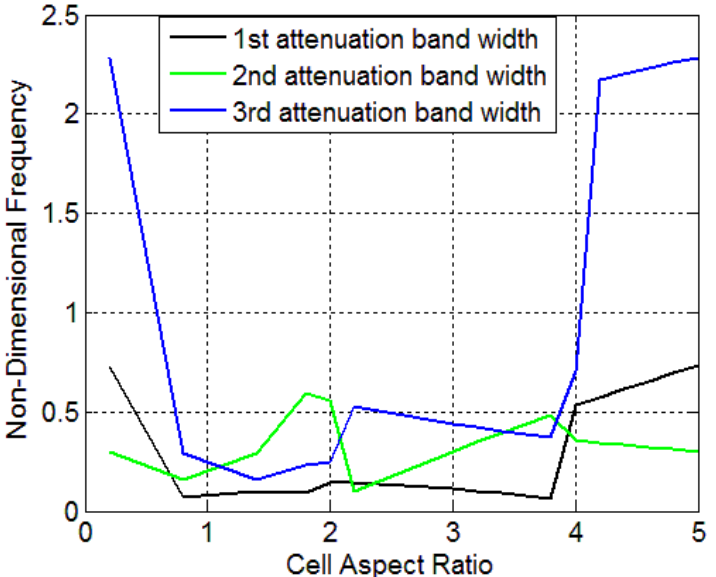

Figure $231^{\text {st }}, 2^{\text {nd }}$ and $3^{\text {rd }}$ stop band width variations with length ratio of cell configuration \#3 at angle $45^{\circ}$ for propagation factor

$$
\mu=\alpha+i \beta
$$

\section{Effect of changing the thickness ratio on the structural dynamic behavior of configuration cell \#4}

Figure 24 shows the last cell configuration to be studied in this investigation. Figure 25 shows that as the thickness ratio approaches 1 the attenuation bands become very small. But elsewhere, the attenuation bands become wider and are shifted to higher frequencies as shown in Figure 25 and Figure 26.

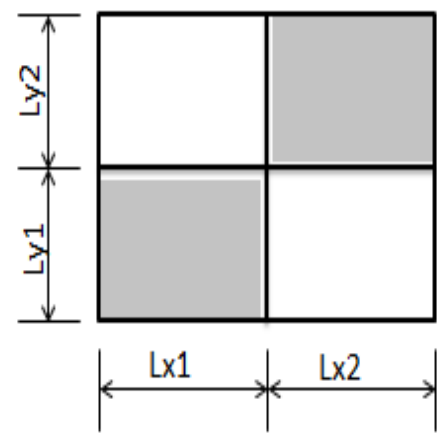

Figure 24 Cell configuration \#4 


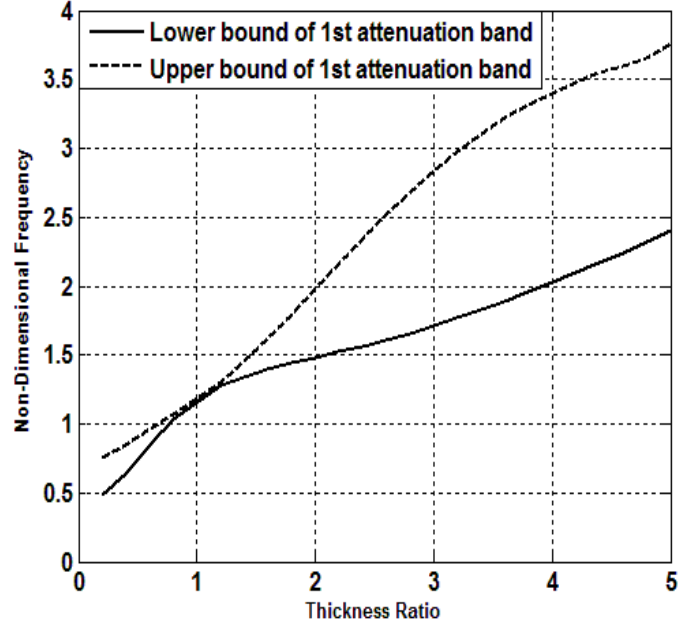

Figure 25 Lower and upper bounds variation with thickness ratio of cell configuration \#4 at angle $45^{\circ}$

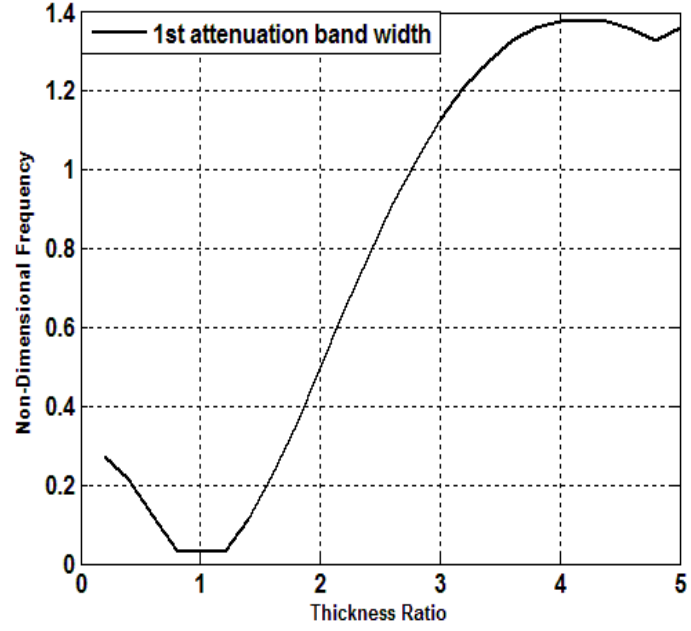

Figure $261^{\text {st }}$ stop band width variations with thickness ratio of cell configuration $\# 3$ at angle $45^{\circ}$

\section{Effect of changing aspect ratio of configuration cell \#4}

Figure 27 shows the start and end of the first, second and third attenuation bands at different aspect ratios for cell configuration \#4 with propagation factor $\mu=\alpha+0$. It shows that at aspect ratio 3.2 the first attenuation band separates into two attenuation bands to form the first and second attenuation bands.

Figure 28 shows the start and end of the first, second, and third attenuation bands for cell configuration \#4 at propagation factor $\mu=\alpha+i \beta$ for different aspect ratios are. It shows that as the aspect ratio increases first attenuation band become smaller until it vanishes at ratio 2.4 to make the second band act as the first band, but it finally appears again at the ratio of 4.8.

Figure 29 and Figure 30 show how the attenuation band widths vary with the variation of aspect ratio for cell configuration \#4 at propagation factors $\mu=\alpha+0$ and $\mu=\alpha+i \beta$.

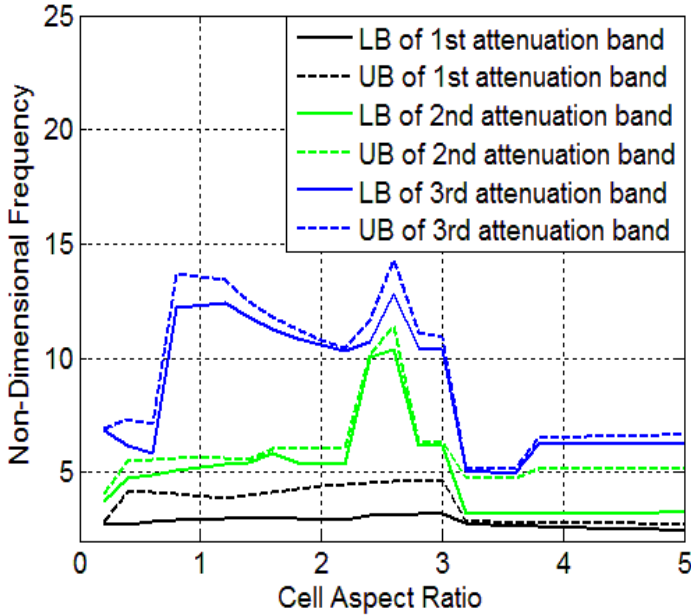

Figure 27 Lower and upper bounds variation with aspect ratio of cell configuration \#4 at angle $45^{\circ}$ for propagation factor $\mu=\alpha+0$

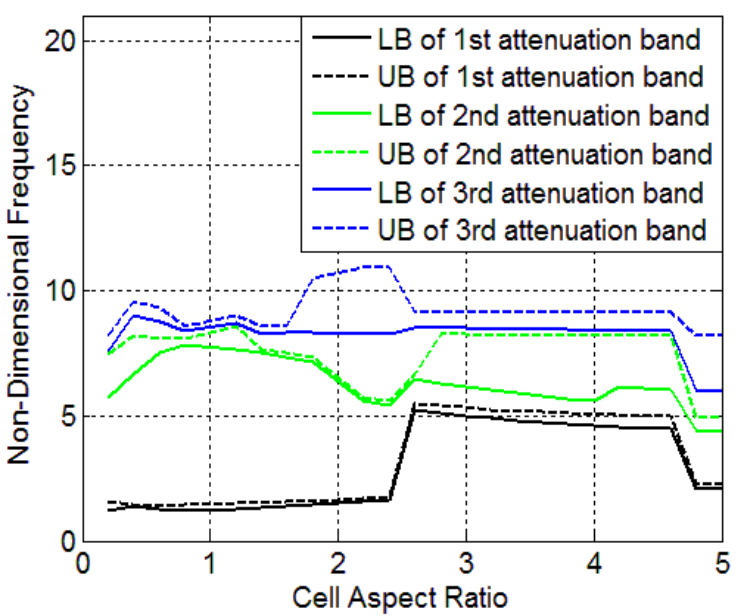

Figure 28 Lower and upper bounds variation with aspect ratio of cell configuration \#4 at angle $45^{\circ}$ for propagation factor $\mu=\alpha+i \beta$ 


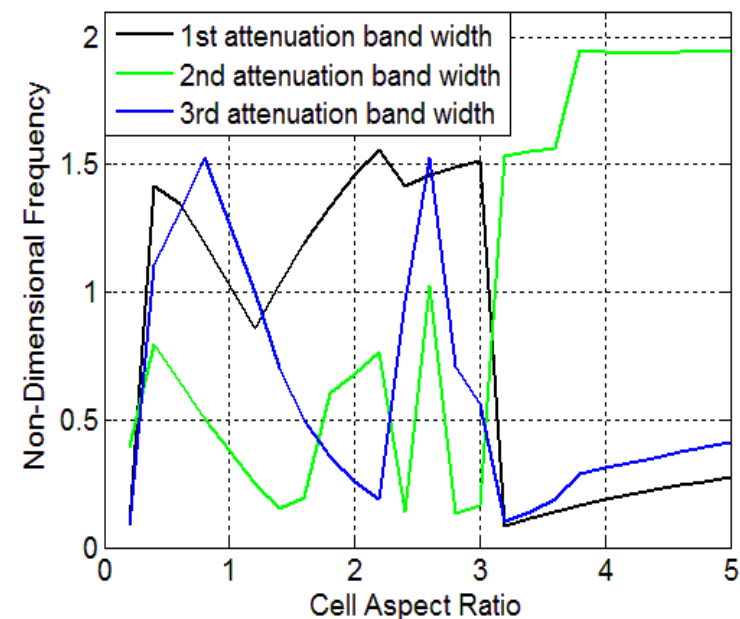

Figure $291^{\text {st }}, 2^{\text {nd }}$ and $3^{\text {rd }}$ stop band width variations with aspect ratio of cell configuration \#4 at angle $45^{\circ}$ for propagation factor

$$
\boldsymbol{\mu}=\boldsymbol{\alpha}+\mathbf{0}
$$

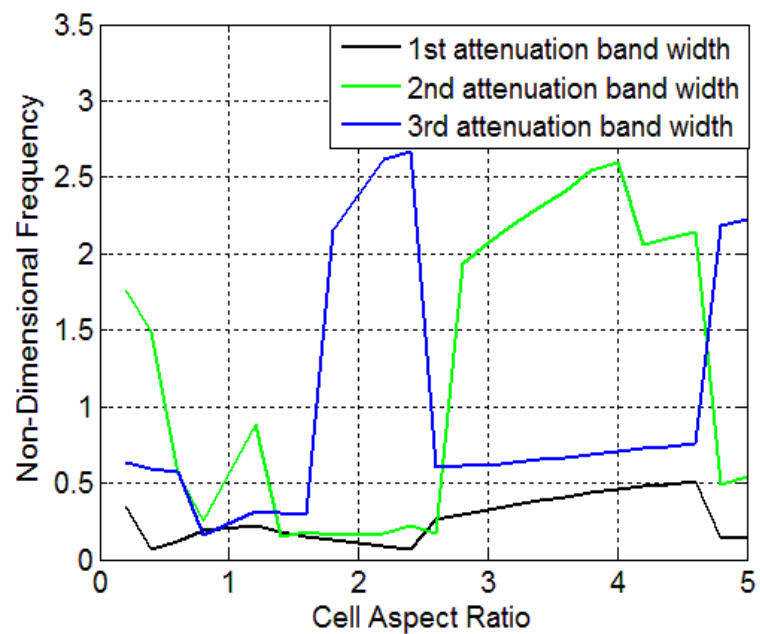

Figure $301^{\text {st }}, 2^{\text {nd }}$ and $3^{\text {rd }}$ stop band width variations with aspect ratio of cell configuration \#4 at angle $45^{\circ}$ for propagation factor $\mu=\alpha+i \beta$

\section{Conclusion}

A study of how the bands of vibration attenuation are affected by changing the plate periodicity configuration, thickness and aspect ratio is described in this chapter. From this study it is shown that for different configurations the variation of thickness ratio has an obvious direct effect on the location and width of the attenuation bands. We may say that above thickness ratio 1 as the thickness ratio increases the attenuation bands are shifted to higher frequencies and become wider. When the thickness ratio decreases below 1 the attenuation bands shift to higher frequencies and become wider. Variation of the aspect ratio has also an effect on the location and width of the attenuation bands, but there is no clear trend for this effect.

\section{References}

[1] Tsung-Tsong Wu, Zi-Gui Tsai, Tzung-Chen Wu, "Evidence of complete band gap and resonances in a plate with periodic stubbed surface,"Taiwan: APPLIED PHYSICS LETTERS 93, 2008.

[2] F Casadei, M Ruzzene, LDozio, K A Cunefare, "Broadband vibration control through periodic arrays of resonanat shunts: experimental investigation on plates", Smart Materials and Structures. stacks.iop.org/SMS/19/015002. [Online] November 19, 2009.

[3] AboElsooud, Mohammad Tawfik, "Vibration control of plates using periodically disributed shunted piezoelectric patches", University of Maryland : s.n., 2003.

[4] Filippo Casadei, Lorenzo Dozio, Massimo Ruzzene, Kenneth A. Cunefare, "Periodic shunted arrayes for the control of noise radiation in an enclosure", Journal of Sound and Vibration, p. 15. 2010.

[5] Yeung, Yum-Tong Siu and Sai-Kee, "A generalized Bloch's theorem and the hyperbolicity of the complement of an ample divisor in an Abelian variety", USA : Mathematics Subject Classification, 1991.

[6] Diperna, Daniel T/ Blake, William K/ Diperna, Xingguang Z., "Computational of the homogeneous and forced solutions of a finite length, line-driven, submerged plate”, The Journal of the Acoustical Society of America. 2006. 
[7] W. Xiao, G.W. zeng, Y. S. Cheng, "Flexural vibration band gaps in a thin plate containing a periodic array of hemmed discs", ELSEVIER, p. 7. 2006.

[8] J.B. Casimir, S. Kevorkian, T. Vinh. . J.B. Casimir, S. Kevorkian, T. Vinh, "The dynamic stiffness matrix of two-dimensional elements: application to Kirchhoff's plate continuous elements", France, Journal of Sound and Vibration , 2005.

[9] Hull, Andrew J., "Dynamic response of an elastic plate containing periodic masses", ELSEVIER, p. 20. 2007.

[10] O. A. Ganilova, M.P.Cartmell, "An analytical model for the vibration of a composite plate containing an embedded periodic shape memory alloy structure", Elsevier, 2009.

[11] S.A Nazarov, G.H.Sweers, A.S.Slutskii, "The flexural rigidity of a thin plate reinforced with periodic systems of separated rods", Journal of Applied Mathematics and Mechanics, p. 10. 2008.

[12] Somaia Talaat, Ahmed Desoki, Mohammad Tawfik, Hani M. Negm, "Experimental and Numerical Investigation of the Capabilities of Periodic Plates for Vibration Attenuation", AMME-15, Cairo, Egypt. May 2012 . 